\title{
Stored blood: how old is too old?
}

\author{
Janet S. Lee ${ }^{1,2}$ and Daniel B. Kim-Shapiro ${ }^{3,4}$ \\ 'Vascular Medicine Institute and 2Department of Medicine, Division of Pulmonary, Allergy and Critical Care Medicine, University of Pittsburgh, Pittsburgh, Pennsylvania, USA. \\ ${ }^{3}$ Department of Physics and ${ }^{4}$ Translational Science Center, Wake Forest University, Winston-Salem, North Carolina, USA.
}

Transfusion of rbc is a routine, often lifesaving procedure that depends on a stored supply of blood. In the US, 42 days is the maximum duration allowed for rbc storage; however, several lines of evidence indicate that patients that receive blood at the upper end of this storage limit are at a higher risk of morbidity and mortality. In this issue of the $J C I$, Rapido and colleagues evaluated the effects of transfusing one unit of blood close to the storage limit into healthy adults. Compared to those that received rbc stored for five weeks or less, those that received blood stored for six weeks showed several effects associated with increased harm, including disruption in iron handling, increased extravascular hemolysis, and the formation of circulating non-transferrin-bound iron. Together, the results of this study suggest that current maximum storage durations should be carefully reevaluated.

\section{Storage of rbc: a controversial necessity}

Transfusion of rbc is a common practice in medicine and is one of the therapies on the World Health Organization's Model List of Essential Medicines (1). During cold storage, rbc undergo senescence that involves a series of biochemical, biophysical, and metabolic changes that are collectively termed "storage lesion." There has been substantial controversy regarding the in vivo consequences of the rbc storage lesion and whether these changes affect physiological end points or clinical outcomes, particularly with regard to cells that have been stored for times near the end of the acceptable storage period. In the US, the shelf life for rbc in refrigerated storage is up to 42 days. The FDA requires that rbc units show less than $1 \%$ hemolysis at the end of storage and that the average 24 -hour posttransfusion recovery of radiolabeled autologous rbc in healthy volunteers in circulation is $75 \%$ or more (2). The performance standard for rbc units has changed little since its original description in 1947 in JCI by Ross et al. (3). A distinct limitation of this standard is that physiologic end points or clinical outcomes of transfusion recipients are not addressed, and this standard may require reappraisal in the modern age. In this issue, Rapido et al. provide evidence that challenges the current maximal allowable storage period (4).

Although the life-saving attributes of rbc transfusion are unequivocal, numerous experimental animal and observational clinical studies have suggested that transfusions, particularly of rbc at the limits of storage time, are associated with increased morbidity and mortality. In 2008, a single center retrospective analysis of over 5,000 patients showed a striking association between increased duration of rbc storage and complications following cardiac surgery that spurred the international scientific community to conduct more than 13 randomized controlled trials (RCT) to formally test this association (5). Collectively, these studies showed that transfusion of

\section{Related Article: p. 375}

Conflict of interest: D.B. Kim-Shapiro is a coinventor on a patent entitled "Methods of treatment for hemolysis" (US patent number $8,980,871$ )

Reference information: J Clin Invest. 2017;127(1):100-102. doi:10.1172/JC191309.

fresh $r b c$ was not superior to that of older stored rbc; however, these conclusions came with a caveat (6-8). None of these RCTs were designed to address the effects of a prolonged storage duration (35-42 days). Given the ethical quandary of randomizing individuals to an arm that would require those subjects to receive blood that has been aged to the limits of acceptable duration, a prospective RCT will never be conducted to address the question of very old blood. Yet the question of how old is too old remains relevant, as Rapido and colleagues note that $9 \%$ to $20 \%$ of standard issue rbc units in some US centers are 35 or more days old (9). Moreover, a recent retrospective analysis of over 13,000 patients showed that critically ill patients transfused exclusively with rbc units stored for 35 days or more, but not those transfused with rbc units stored for 28 to 34 days, exhibited increased morbidity and mortality compared with those that received rbc units stored for 21 days or less (10).

\section{Adverse effects of a single unit of aged blood}

Rapido and colleagues examined the outcomes associated with transfusion of a single unit of autologous blood (packed rbc) after 1 to 6 weeks of storage (4). Remarkably, transfusion of just a single unit of 6-week-old packed rbc (35-42 days) overwhelmed normal iron binding capacity via transferrin, resulting in non-transferrin-bound iron (NTBI) (4). The levels of the iron regulatory hormone hepcidin also increased as a function of storage time, indicating that a physiological response aimed at reducing iron export into the plasma was insufficient to avoid formation of NTBI in $78 \%$ of those transfused with one 6-week-old unit. A similar hepcidin increase was also observed in one individual transfused with a 5-week-old unit. Notably, the disruption in iron homeostasis lasted for up to ten to twelve hours. The percent recovery of rbc was observed to decrease with increasing storage time with 6-week blood resulting in less than $75 \%$ 


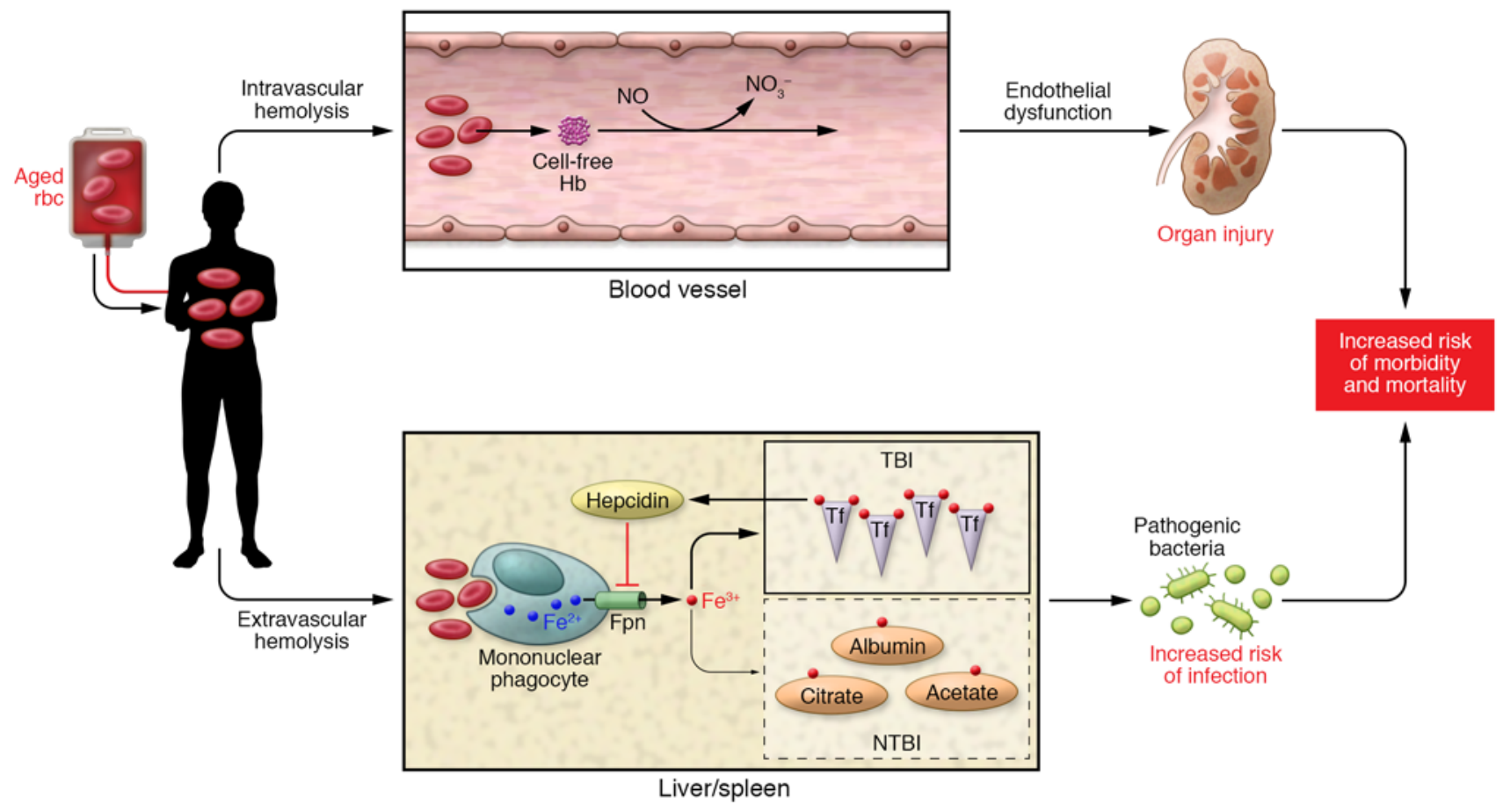

Figure 1. Schematic representation of aged rbc transfusion effects. Intravascular hemolysis occurs, resulting in the release of breakdown products from aged rbc and cell-free hemoglobin $(\mathrm{Hb}$, represented by cell-free $\mathrm{Hb}$ ). Additionally, scavenging of NO occurs through the dioxygenation reaction. Release of arginase from lysing rbc also reduces the substrate L-arginine for NO synthase, further reducing NO bioavailability (not depicted). Reduced NO decreases vessel smooth muscle relaxation and contributes to endothelial dysfunction, with consequent organ injury. Extravascular hemolysis occurs when damaged rbc are acutely delivered and exceed the capacity of the mononuclear phagocyte system of the liver and spleen. Aged rbc are phagocytosed, heme is catabolized, and iron is ultimately released through the ferroportin (Fpn) export system and taken up by transferrin (Tf), the major iron transport protein in the body. With aged rbc transfusion, the large amount of iron exported out of the monocyte/macrophage results in the development of NTBI - iron complexed to other compounds such as albumin, citrate, etc. The disruption in iron handling may provide a readily available source of iron for pathogens and increase infectious risk in susceptible hosts. Note that a rise in transferrin-bound iron also occurs and provides a feedback loop to increase hepcidin, which negatively regulates iron export through ferroportin. However, this compensatory mechanism is insufficient to avoid formation of NTBI. For simplicity, only key elements of the two pathways are depicted.

recovery in one case. Moreover, both the increase in iron and decrease in rbc recovery were attributed to extravascular hemolysis, based on the observation that indirect (unconjugated) bilirubin also increased as a function of storage age, whereas markers of intravascular hemolysis (plasma hemoglobin, lactate dehydrogenase, and haptoglobin levels) did not change.

Although Rapido et al. did not provide evidence to support intravascular hemolysis, a recent study by Risbano et al. showed otherwise following transfusion of older blood (11). Risbano et al. infused half a unit of autologous blood that had been stored for 5 or 42 days into the forearm brachial arteries of healthy volunteers. Venous blood was collected in the same arm to minimize systemic dilution. These local measurements were used to mimic what would happen systemically in a larger transfusion. Plasma cell-free hemoglobin increased due to transfusion of 42-dayold blood. Levels of arginase 1 (which is released from red blood cells and catalyzes the conversion of the NO synthase substrate arginine to ornithine) and lactate dehydrogenase also increased. Intravascular hemolysis was associated with increased NO scavenging and impaired endothelial function after transfusion of 42-day-old blood. However, in the study by Rapido et al., markers of intravascular hemolysis were reduced below detection levels, likely due to systemic dilution.

Both intravascular and extravascular hemolysis have been postulated to mediate harm through endothelial dysfunction and production of NTBI, respectively (Figure 1). NTBI may increase the virulence of bloodstream pathogens in "susceptible" patients in intensive care units already predisposed to infectious risk (12). Rapido et al. observed a substantial amount of NTBI after transfusion of 6-week-old stored rbc when only one unit was transfused (4). Would NTBI have been detected in volunteers after larger transfusions of blood stored for fewer weeks? Transfusion of one unit of 4-week-old and 5-week-old stored blood resulted in transferrin saturation nearing 50\% (4). Thus, one might think that transfusion of two or three units of 4- or 5-week-old stored rbc would lead to NTBI accumulation. This analysis suggests that risk of NTBI production would be a function of the age of the units as well as the number of those units.

\section{Conclusions and future considerations}

More work is needed to address some basic questions about the distribution of different forms of iron in the plasma and their potential for harm. The equilibrium binding affinity of ferric iron to transferrin is extremely high $\left(K=1.6 \times 10^{22} \mathrm{M}^{-1}\right.$; ref. 13$)$. Based solely on this extremely high affinity, even when transferrin is $99.9 \%$ saturated, the concentration of NTBI would still be less than a billionth of a nanomolar. However, Rapido et al. detected NTBI in 
the micromolar range even at much lower transferrin saturations (4). This observation may seem surprising, but NTBI can be detected even at transferrin saturations as low as $50 \%$ to $60 \%$ (14). The NTBI is distributed among plasma proteins (including albumin), citrate, and perhaps acetate, malate, and phosphate $(15,16)$. The distribution of iron among transferrin, citrate, albumin, and other binders likely depends largely on kinetics of transfer to transferrin, which has been found to be slow and complicated under certain conditions (17). Transferrin bound to iron is thought to be relatively benign in terms of promoting oxidative stress (18). Determining the forms that are most prevalent under different conditions and their relative potential for harm requires additional study, although a recent report indicates that iron citrate has redox activity (19).

Based on their findings, Rapido and colleagues suggest that the FDA should consider changing the maximum storage age of rbc to 35 days instead of 42 days, as the $\mathrm{NIH}$ and some countries have already done (4, 20-22). Such consideration should include the extent to which the major outcome in this study, NTBI, is clinically relevant. As pointed out by Rapido et al., the extent of deleterious NTBI-associated effects may depend on the degree of illness of the rbc recipient. It is also unclear whether a rise in NTBI is an indicator of a more pervasive impairment in macrophage functional capacity following transfusion of rbc near the limits of allowable storage duration. As shown recently by Theurl et al., inhibiting the ability of the mononuclear phagocyte population to adequately clear damaged rbc during stressed or on-demand delivery led to liver and kidney damage in mice (23). Moreover, many bacteria utilize siderophores that have a higher affinity for ferric iron than mammalian proteins (24). Although hosts have coevolved to counter aggressive measures employed by pathogens for iron acquisition, a rise in transferrin-bound iron following transfusion of rbc at the extremes of storage duration may also be harmful. Indeed, host catechols, such as norepinephrine, can liberate iron complexed to transferrin and lactoferrin and provide readily available iron to bacteria (25). As discussed above, it will be important to establish NTBI release, as well as other markers of potential harm, to evaluate transfusion outcomes when more than one unit is infused. In such cases, 5-week-old blood may also have deleterious effects. It is also unknown whether chronic infusion of older units of stored blood leads to an accumulation of adverse effects. The potential benefit of reducing the maximum storage duration time to 35 days must be carefully considered and balanced against potential risks and the costs of reducing the available rbc supply in high-demand regions. One alternative (or additional solution) to shortening the storage time would be to find additives to the storage solution or novel storage methods that would decrease potential deleterious effects. At a minimum, the disruption in iron homeostasis shown by Rapido et al. in healthy adults following transfusion of rbc at the current limits of storage duration highlights the continuing need for further work into how old is too old.

\section{Acknowledgments}

This work was supported by NIH grants HL058091 and HL098032 (to DBKS) and HL086884 and AI119042 (to JSL).

Address correspondence to: Daniel B. Kim-Shapiro, Department of Physics, Wake Forest University, Winston-Salem, North Carolina 27109, USA. Phone: 336.758.4993; E-mail: shapiro@wfu.edu. Or to: Janet S. Lee, Department of Medicine, Division of Pulmonary, Allergy and Critical Care Medicine, University of Pittsburgh, Pittsburgh, Pennsylvania 15213, USA. Phone: 412.692.2210; E-mail: leejs3@upmc.edu.

1. World Health Organization. WHO Model List of Essential Medicines. WHO Web site. http:// www.who.int/medicines/publications/essentialmedicines/en/. Updated November 1, 2015. Accessed November 8, 2016.

2. Dumont LJ, AuBuchon JP. Evaluation of proposed FDA criteria for the evaluation of radiolabeled red cell recovery trials. Transfusion. 2008;48(6):1053-1060.

3. Ross JF, Finch CA, Peacock WC, Sammons ME. The in vitro preservation and post-transfusion survival of stored blood. JClin Invest. 1947;26(4):687-703.

4. Rapido F, et al. Prolonged red cell storage before transfusion increases extravascular hemolysis. J Clin Invest. 2017;127(1):375-382.

5. Koch CG, et al. Duration of red-cell storage and complications after cardiac surgery. $N$ Engl J Med. 2008;358(12):1229-1239.

6. Lacroix J, et al. Age of transfused blood in critically ill adults. N Engl JMed. 2015;372(15):1410-1418.

7. Steiner ME, et al. Effects of red-cell storage duration on patients undergoing cardiac surgery. N Engl JMed. 2015;372(15):1419-1429.

8. Heddle NM, et al. Effect of short-term vs. long-term blood storage on mortality after transfusion [published online ahead of print October 24, 2016]. NEngl JMed. doi:10.1056/ NEJMoa1609014.

9. Glynn SA, Klein HG, Ness PM. The red blood cell storage lesion: the end of the beginning. Transfusion. 2016;56(6):1462-1468.

10. Goel R, et al. Red blood cells stored 35 days or more are associated with adverse outcomes in high-risk patients. Transfusion. 2016;56(7):1690-1698.

11. Risbano MG, et al. Effects of aged stored autologous red blood cells on human endothelial function. Am J Resp Crit Care. 2015;192(10):1223-1233.

12. Hod EA, Spitalnik SL. Stored red blood cell transfusions: Iron, inflammation, immunity, and infection. Transfu Clin Biol. 2012;19(3):84-89.

13. Aisen P, Listowsky I. Iron transport and storage proteins. Annu Rev Biochem. 1980;49:357-393.

14. Vanderheul C, Vaneijk HG, Wiltink WF, Leijnse B. Binding of iron to transferrin and to other serum components at different degrees of saturation with iron. Clin Chim Acta. 1972;38(2):347-353.

15. Hider RC. Nature of nontransferrin-bound iron. Eur J Clin Invest. 2002;32(suppl 1):50-54.

16. de Swart L, et al. Second international round robin for the quantification of serum non-transferrin-bound iron and labile plasma iron in patients with iron-overload disorders. Haematologica. 2016;101(1):38-45.

17. Bates GW, Billups C, Saltman P. The kinetics and mechanism of iron (3) exchange between chelates and transferrin. I. The complexes of citrate and nitrilotriacetic acid. J Biol Chem. 1967;242(12):2810-2815.

18. Elsayed ME, Sharif MU, Stack AG. Transferrin saturation: a body iron biomarker. Adv Clin Chem. 2016;75:71-97.

19. Adam FI, Bounds PL, Kissner R, Koppenol WH. Redox properties and activity of iron-citrate complexes: evidence for redox cycling. Chem Res Toxicol. 2015;28(4):604-614.

20. Dzik WH, et al. Factors affecting red blood cell storage age at the time of transfusion. Transfusion. 2013;53(12):3110-3119.

21. Blake JT, Hardy M, Delage G, Myhal G. Deja-vu all over again: using simulation to evaluate the impact of shorter shelf life for red blood cells at Hema-Quebec. Transfusion. 2013;53(7):1544-1558.

22. Klein HG, Cortes-Puch I, Natanson C. More on the age of transfused red cells. N Engl J Med. 2015;373(3):283.

23. Theurl I, et al. On-demand erythrocyte disposal and iron recycling requires transient macrophages in the liver. Nat Med. 2016;22(8):945-951.

24. Wooldridge KG, Williams PH. Iron uptake mechanisms of pathogenic bacteria. FEMS Microbiol Rev. 1993;12(4):325-348.

25. Sandrini SM, et al. Elucidation of the mechanism by which catecholamine stress hormones liberate iron from the innate immune defense proteins transferrin and lactoferrin. J Bacteriol. 2010;192(2):587-594. 